\title{
A Data Set Oriented Approach for Clustering Algorithm Selection
}

\author{
Maria Halkich and Michalis Vazirgiannis \\ Department of Informatics \\ Athens University of Economics \& Business \\ Patision 76, 10434, Athens, Greece (Hellas) \\ \{mhalk, mvazirg\}@aueb.gr
}

\begin{abstract}
In the last years the availability of huge transactional and experimental data sets and the arising requirements for data mining created needs for clustering algorithms that scale and can be applied in diverse domains. Thus, a variety of algorithms have been proposed which have application in different fields and may result in different partitioning of a data set, depending on the specific clustering criterion used. Moreover, since clustering is an unsupervised process, most of the algorithms are based on assumptions in order to define a partitioning of a data set. It is then obvious that in most applications the final clustering scheme requires some sort of evaluation.

In this paper we present a clustering validity procedure, which taking in account the inherent features of a data set evaluates the results of different clustering algorithms applied to it. A validity index, $S \_D b w$, is defined according to wellknown clustering criteria so as to enable the selection of the algorithm providing the best partitioning of a data set. We evaluate the reliability of our approach both theoretically and experimentally, considering three representative clustering algorithms ran on synthetic and real data sets. It performed favorably in all studies, giving an indication of the algorithm that is suitable for the considered application.
\end{abstract}

\section{$1 \quad$ Introduction \& Motivation}

Clustering is one of the most useful tasks in data mining process for discovering groups and identifying interesting distributions and patterns in the underlying data. Thus, the main concern in the clustering process is to reveal the organization of patterns into "sensible" groups, which allow us to discover similarities and differences, as well as to derive useful inferences about them [7].

In the literature a wide variety of algorithms have been proposed for different applications and sizes of data sets [14]. The application of an algorithm to a data set aims at, assuming that the data set offers such a clustering tendency, discovering its inherent partitions. However, the clustering process is perceived as an unsupervised process, since there are no predefined classes and no examples that would show what kind of desirable relations should be valid among the data [2]. Then, the various clustering algorithms are based on some assumptions in order to define a partitioning of a data set. As a consequence, they may behave in a different way depending on: i) the fea- 


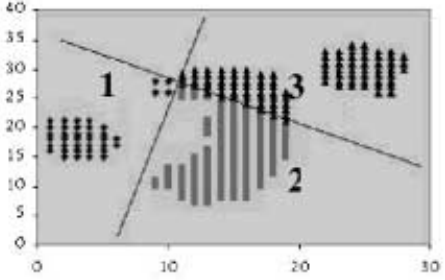

(a)

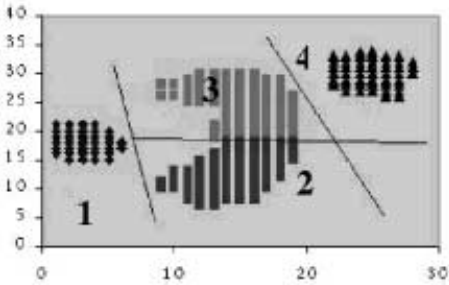

(b)

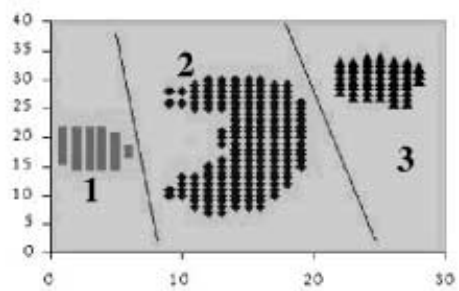

(c)

Fig. 1. A two dimensional data set partitioned into (a) three and (b) four clusters using KMeans (c) three clusters using DBSCAN
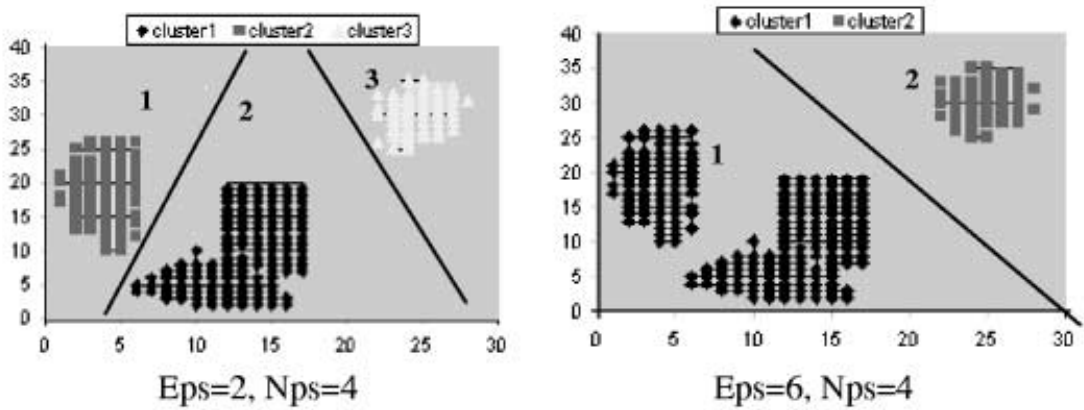

Fig. 2. The different partitions resulting from running DBSCAN with different input parameter values

tures of the data set (geometry and density distribution of clusters) and ii) the input parameters values.

Partitional algorithms such as K-means [2] are unable to handle noise and outliers and they are not suitable to discover clusters with non-convex shapes. Moreover, they are based on certain assumptions to partition a data set. They need to specify the number of clusters in advance except for CLARANS [11], which needs as input the maximum number of neighbors of a node as well as the number of local minima that will be found in order to define a partitioning of a data set. Also, hierarchical algorithms [9] proceed successively by either merging smaller clusters into larger ones or 
by splitting larger clusters. The result of these algorithms is a tree of clusters. Depending on the level at which we cut the tree, a different clustering of the data is obtained. On the other hand density-based [3, 4, 8] and grid-based algorithms [12] suitably handle arbitrary shaped collections of points (e.g. ellipsoidal, spiral, cylindrical) as well as clusters of different sizes. Moreover, they can efficiently separate noise (outliers). However, most of these algorithms are sensitive to some input parameters so as to require careful selection of their values.

It is obvious from above discussion that the final partition of a data set requires some sort of evaluation in most applications [16]. Then, an important issue in clustering is to find out the number of clusters that give the optimal partitioning (i.e, the partitioning that best fits the real partitions of the data set). Though this is an important problem that causes much discussion, the formal methods for finding the optimal number of clusters in a data set are few $[10,13,14,15]$. Moreover, all clustering algorithms are not efficient for all applications, which is why a diverse of algorithms has been developed. Depending on the clustering criterion and the ability to handle the special requirements of an application a clustering algorithm can be considered more efficient in a certain context (e.g. spatial data, business, medicine etc). However, the issue of cluster validity is rather under-addressed in the area of databases and data mining applications, while there are no efforts regarding the evaluation of clustering schemes defined by different clustering algorithms.

Further more, in most algorithms' experimental evaluations $[1,4,6,7,8,11] 2 \mathrm{D}$ data sets are used in order the reader is able to visually verify the validity of the results (i.e., how well the clustering algorithm discovered the clusters of the data set). It is clear that visualization of the data set is a crucial verification of the clustering results. In the case of large multidimensional data sets (e.g. more than three dimensions) effective visualization of the data set can be difficult. Moreover the perception of clusters using available visualization tools is a difficult task for the humans that are not accustomed to higher dimensional spaces.

Assuming that the data set includes distinct partitions (i.e., inherently supports clustering tendency), the above issues become very important. In the sequel, we show that different input parameters values of clustering algorithms may result in good or bad results in partitioning the data set. Moreover, it is clear that some algorithms may fail to discover the actual partitioning of a data set though the correct number of clusters is considered.

For instance in Fig. 1 and Fig. 2 we can see the way different algorithms (DBSCAN [4], K-Means [2]) partition a data set having different input parameter values. Moreover, it is clear from Fig. 1a that K-means may partition the data set into the correct number of clusters (i.e., three clusters) but in a wrong way. On the other hand,

DBSCAN (see Fig. 1c) is more efficient since it partitioned the data set in the inherent three clusters under the consideration of the suitable input parameters' values. As it is evident, if there is no visual perception of the clusters it is impossible to assess the validity of the partitioning. It is important then to be able to choose the optimal partitioning of a data set as a result of applying different algorithms with different input parameter values.

What is then needed is a visual-aids-free assessment of some objective criterion, indicating the validity of the results of a clustering algorithm application on a potentially high dimensional data set. In this paper we propose an evaluation procedure 
based on a cluster validity index (S_Dbw). Assuming a data set S, the index enables the selection of the clustering algorithm and its input parameter values so as to result in the best partitioning of $\mathrm{S}$.

The remainder of the paper is organized as follows. In the next section we motivate and define the validity index, while in Section 3 we provide a theoretical evaluation of S_Dbw based on its definition. Then, in Section 4 an experimental evaluation of our approach for selecting the algorithm that gives the optimal partitioning of a data set is presented. For the experimental study both synthetic and real data sets are used. We conclude in Section 5 by briefly presenting our contributions and indicate directions for further research.

\section{Selecting the Best Algorithm}

One of the most widely studied problems in area of knowledge discovery is the identification of clusters, i.e., dense region in multidimensional data sets. This is also the subject of cluster analysis. Clustering is perceived as a complicated problem since depending on the application domain and the feature of data the concept of cluster may be different. Thus to satisfy the requirements of diverse application domains, a multitude of clustering methods are developed and are available in the literature. However, the problem of selecting the algorithm that may result in the partitioning that best fits a data set is under-addressed. In the sequel, we present our approach and we define the validity index based on which we may evaluate the clustering schemes under consideration and select the one best fits the data.

\subsection{An Approach of Clustering Schemes' Evaluation}

The criteria widely accepted for evaluating the partitioning a data set are: i. the separation of the clusters, and ii. their compactness. In general terms we want clusters whose members have a high degree of similarity (or in geometrical terms are close to each other) while we want the clusters themselves to be widely spread.

As we have already mentioned, there are cases that an algorithm may falsely partition a data set, whereas only specific values for the algorithms' input parameters lead to optimal partitioning of the data set. Here the term "optimal" implies parameters that lead to partitions that are as close as possible (in terms of similarity) to the actual partitions of the data set.

Then our objective is the definition of a procedure for assessing the quality of partitioning as defined by a number of clustering algorithms. More specifically, a validity index is defined which based on the features of the clusters may evaluate the resulting clustering schemes as defined by the algorithm under consideration. Then, the algorithm and the respective set of input parameters resulting in the optimal partitioning of a data set may be selected.

Let a data set $\mathrm{S}$ and $\mathrm{Alg}=\left\{\operatorname{alg}_{\mathrm{i}} \mid \mathrm{i}=1, . ., \mathrm{k}\right\}$ a set of widely known clustering algorithms. $\mathrm{Palg}_{i}$ denotes the set of input parameters of an algorithm, alg $_{i}$. Applying a clustering algorithm $\operatorname{alg}_{i}$ to $\mathrm{S}$ while their parameters take values in $\mathrm{Palg}_{i}$ a set of clustering schemes is defined, let $\mathrm{Calg}_{\mathrm{i}}=\left\{\mathrm{c}_{\mathrm{p} \_} \mathrm{alg}_{\mathrm{i}} \mid \mathrm{p} \in \mathrm{Palg}_{\mathrm{i}}\right\}$. Then, the clustering schemes are evaluated based on a validity index we define, S_Dbw. A list of the index values is 
maintained, denoted as index, for each set Calg $\mathrm{g}_{\mathrm{i}}$ defined by the available algorithms. The main steps of our approach can be described as follows:

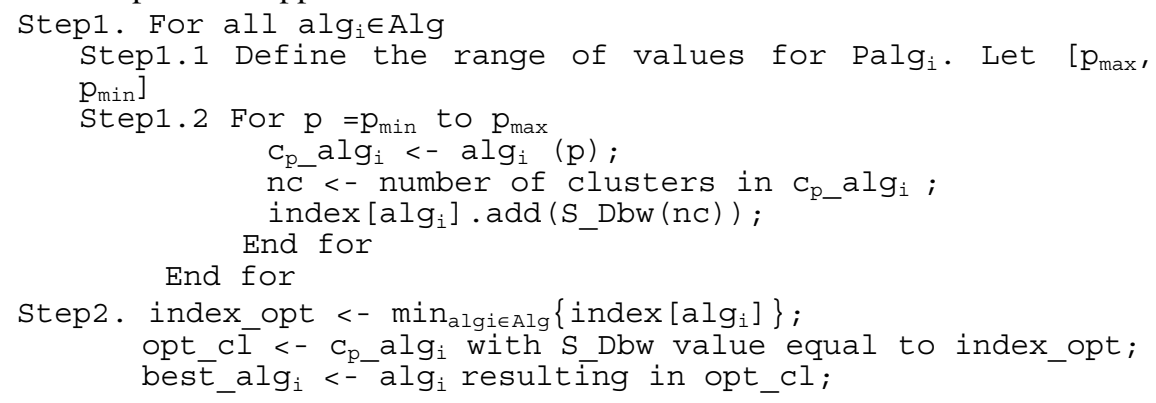

In the sequel, we discuss in more detail the validity index which our approach use in order to select the clustering algorithm resulting in optimal partitioning of a data set.

\subsection{Validity Index Definition}

We define our validity index, S_Dbw, combining both clustering criteria (compactness and separation) taking also in account density. In the following we formalize our clustering validity index based on: i. clusters' compactness (in terms of intra-cluster variance), and ii. density between clusters (in terms of inter-cluster density).

Let $D=\left\{v_{i} l i=1, \ldots, c\right\}$ a partitioning of a data set $S$ into $c$ clusters where $v_{i}$ is the center of $\mathrm{i}$ cluster as it results from applying a clustering algorithm $\operatorname{alg}_{j}$ to $\mathrm{S}$.

Let stdev the average standard deviation of clusters defined as: $\quad$ stdev $=\frac{1}{c} \sqrt{!_{i=1}^{c}\left\|\sigma\left(v_{i}\right)\right\|} \quad 1$

Then the overall inter-cluster density is defined as:

Definition 1. Inter-cluster Density (ID) - It evaluates the average density in the region among clusters in relation with the density of the clusters. The goal is the density among clusters to be significant low in comparison with the density in the considered clusters. Then, we can define inter-cluster density as follows:

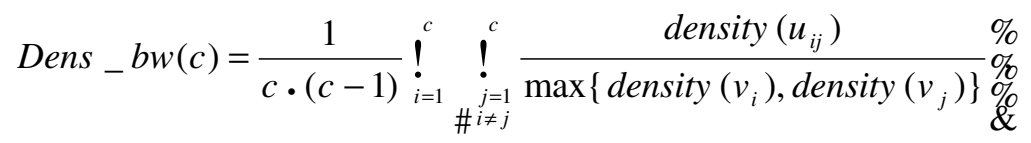

where $v_{i}, v_{j}$ centers of clusters $c_{i}, c_{j}$, respectively and $u_{i j}$ the middle point of the line segment defined by the clusters' centers $v_{i}, v_{j}$. The term density $(u)$ is given by equation

$$
\operatorname{density}(u)=!_{i=1}^{n_{i j}} f\left(x_{l}, u\right)
$$

where $n_{i j}=$ number of tuples that belong to the clusters $\mathrm{c}_{\mathrm{i}}$ and $\mathrm{c}_{\mathrm{j}}$, i.e., $x_{l} \in c_{i} \cup c_{j} \subseteq S$

\footnotetext{
${ }^{1}$ The term $\|x\|$ is defined as : $\|x\|=\left(x^{T} x\right)^{1 / 2}$, where $d$ dimension of $x$ vector.
} 
represents the number of points in the neighbourhood of $u$. In our work, the neighbourhood of a data point, $u$, is defined to be a hyper sphere with center $u$ and radius the average standard deviation of the clusters, stdev. More specifically, the function $f(x, u)$ is defined as:

$$
f(x, u)= \begin{cases}0, & \text { if } \mathrm{d}(\mathrm{x}, \mathrm{u})>\text { stdev } \\ 1, & \text { otherwise }\end{cases}
$$

It is obvious that a point belongs to the neighborhood of $\mathrm{u}$ if its distance from $\mathrm{u}$ is smaller than the average standard deviation of clusters. Here we assume that the data have been scaled to consider all dimensions (bringing them into comparable ranges) as equally important during the process of finding the neighbors of a multidimensional point [2].

Definition 2. Intra-cluster variance - Average scattering for clusters. The average scattering for clusters is defined as:

$$
\operatorname{Scat}(c)=\frac{\frac{1}{c} !_{i=1}^{c}\left\|\sigma\left(v_{i}\right)\right\|}{\|\sigma(X)\|}
$$

The term ! (" ) is the variance of a data set; and its $\mathrm{p}_{\mathrm{th}}$ dimension is defined as follows:

$$
\sigma_{x}^{p}=\frac{1}{n} !_{k=1}^{n}\left(x_{k}^{p}-\bar{x}^{p}\right)^{2}
$$

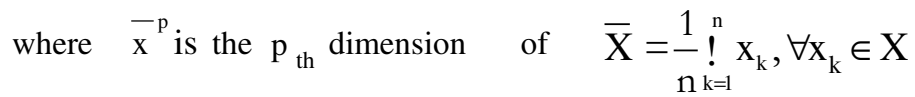

The term ! $\left(\mathrm{v}_{\mathrm{i}}\right)$ is the variance of cluster $\mathrm{c}_{\mathrm{i}}$ and its $\mathrm{p}_{\text {th }}$ dimension is given by

$$
\sigma_{v_{i}}^{p}=!_{k=1}^{n_{i}}\left(x_{k}^{p}-v_{i}^{p}\right)^{2} / n_{i}
$$

Then the validity index S_Dbw is defined as:

$$
\text { S_Dbw }=\text { Scat }(c)+\text { Dens_bw }(c)
$$

The definition of S_Dbw indicates that both criteria of "good" clustering (i.e., compactness and separation) are properly combined, enabling reliable evaluation of clustering results. The first term of S_Dbw, $\operatorname{Scat}(c)$, indicates the average scattering within c clusters. A small value of this term is an indication of compact clusters. As the scattering within clusters increases (i.e., they become less compact) the value of $S c a t(c)$ also increases and therefore it is a good indication of the compactness of clusters. Dens_bw(c) indicates the average number of points between the $c$ clusters (i.e., an indication of inter-cluster density) in relation with density within clusters. A small Dens_bw(c) value indicates well-separated clusters. The number of clusters, c, that minimizes the above index can be considered as an optimal value for the number of clusters present in the data set. 


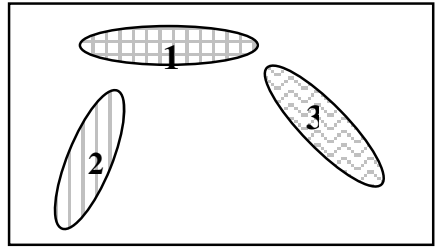

(a)

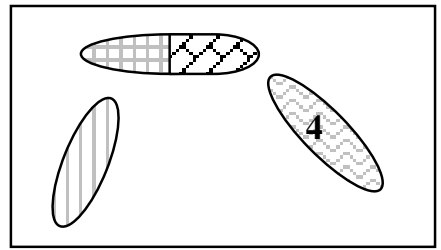

(c)

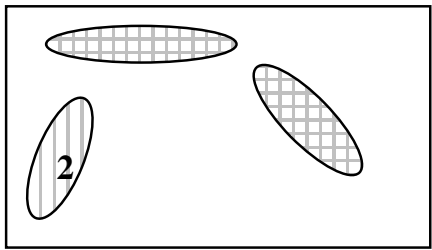

(b)

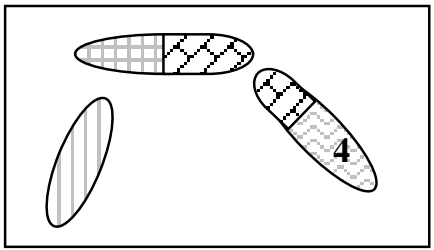

(d)

Fig. 3. A data set $\mathrm{S}$ partitioned in three (a) two (b) and four clusters (c, d)

\section{Integrity Issues}

In this section we evaluate the integrity of the validity index S_Dbw on which our approach based as regards their ability to select the best partitioning among these proposed by the clustering algorithms. In the following lemmas we summarize the different cases of clustering results giving also their respective proof sketches.

Let a data set S containing convex clusters (as in Fig.a) and various ways to partition it using different clustering algorithms (Figure 3b-d). Let the optimal (natural) partitioning of data set $S$ (as it is appeared in Figure 3a) in three clusters. The number of clusters as it emanates from the case of optimal partitioning is further called "correct number of clusters". We assume that the data set is evenly distributed, i.e., on average similar number of data points are found for each surface unit in the clusters.

Lemma 1: Assume a data set $S$ with convex clusters and a clustering algorithm A applied repetitively to $S$, each time with different input parameter values $P_{i}$, resulting in different partitions $D_{i}$ of $S$. The value of $S_{-} D b w$ is minimized when the correct number of clusters is found.

Proof: Let $n$ be the correct number of clusters of the data set $\mathrm{S}$ corresponding to the partitioning $D_{l}$ (optimal partitioning of $\mathrm{S}$ ): $\mathrm{D}_{1}(\mathrm{n}, \mathrm{S})=\left\{\mathrm{c}_{\mathrm{Dl}}\right\}, \mathrm{i}=1, \ldots, \mathrm{n}$

and $m$ the number of clusters of another partitioning $D_{2}$ of the same data set: $\mathrm{D}_{2}(\mathrm{~m}, \mathrm{~S})$ $=\left\{\mathrm{c}_{\mathrm{D} 2 \mathrm{j}}\right\}, \mathrm{j}=1, \ldots, \mathrm{m}$.

Let $S_{-} D b w_{D 1}$ and $S_{-} D b w_{D 2}$ be the values of the validity index for the respective partitioning schemes. Then, we consider the following cases:

i) Assume $D_{2}$ to be a partitioning where more than the actual clusters are formed (i.e., $\mathrm{m}>\mathrm{n}$ ). Moreover, parts of the actual clusters (corresponding to $D_{l}$ ) are grouped into clusters of $D_{2}$ (as in Fig.d). Let $\mathrm{fC}_{\mathrm{D} 1}=\left\{\mathrm{fc}_{\mathrm{Dlp}} \mid \mathrm{p}=1, \ldots, \mathrm{nfr} 1 \mathrm{fc}_{\mathrm{Dlp}} \subseteq \mathrm{c}_{\mathrm{Dli}}, \mathrm{i}=1, \ldots, \mathrm{n}\right\}$ a set of fractions of clusters in $D_{1}$. Similarly, we define $\mathrm{fC}_{\mathrm{D} 2}=\left\{\mathrm{fc}_{\mathrm{D} 2 \mathrm{k}} \mid \mathrm{k}=1, \ldots, \mathrm{nfr} 2\right.$, $\left.\mathrm{fc}_{\mathrm{D} 2 \mathrm{k}} \subseteq \mathrm{c}_{\mathrm{D} 2 \mathrm{j}}, \mathrm{j}=1, \ldots, \mathrm{m}\right\}$. Then: 


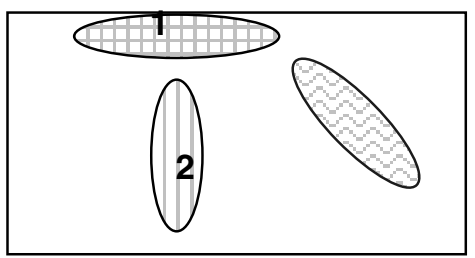

(a)

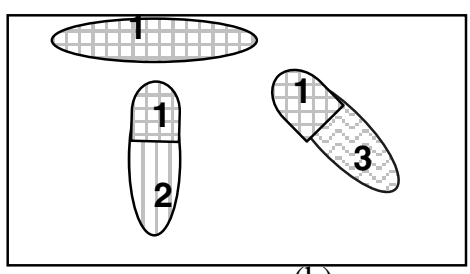

(b)

Fig. 4. A data set S partitioned correctly (a) and falsely (b) in three clusters

a) $\exists c_{D 2 j}: c_{D 2 i}=\cup f_{c_{D 1 p}}$, where $p=p_{1}, \ldots, p_{n}, p_{1}>=1$ and $p_{n}<=n f r 1$, nfr1 is the number of considered fractions of clusters in $\mathrm{D}_{1}$,

b) $\exists c_{D 1 i}: c_{D 1 i}=\cup f c_{D 2 k}$, where $k=k_{1}, \ldots, k_{n}, k_{1}>=1$ and $k_{n}<=$ nfr2, where nfr2 is the number of considered fractions of clusters in $\mathrm{D}_{2}$,

In this case, some of the clusters in $D_{2}$ include regions of low density (for instance cluster 3 in Fig.d). Thus, the value of the first term of the index related to intracluster variance of $D_{2}$ increases as compared to the intra-cluster variance of $D_{1}$ (i.e., Scat $(m)>\operatorname{Scat}(n))$. On the other hand, the second term (inter-cluster density) is also increasing as compared to the corresponding term of index for $D_{l}$ (i.e., Dens_bw(m) > Dens_bw(n)). This is because some of the clusters in $\mathrm{D}_{1}$ are split and therefore there are border areas between clusters that are of high density (e.g., clusters 1 and 3 in Figure 3d). Then, since both S_Dbw terms regarding $D_{2}$ partitioning increase we conclude that $\mathrm{S} \_D b w_{D 1}<S \_D b w_{D 2}$.

ii) Let $D_{2}$ be a partitioning where more clusters than in $D_{l}$ are formed (i.e., $\mathrm{m}>\mathrm{n}$ ). Also, we assume that at least one of the clusters in $D_{l}$ is split to more than one in $\mathrm{D}_{2}$ while no parts of $\mathrm{D}_{1}$ clusters are grouped into $D_{2}$ clusters (as in Fig.c), i.e., $\exists$ $\mathrm{c}_{\mathrm{D} 1 \mathrm{i}}: \mathrm{c}_{\mathrm{D} 1 \mathrm{i}}=\cup \mathrm{c}_{\mathrm{D} 2 \mathrm{j}}, \mathrm{j}=\mathrm{k}_{1}, \ldots, \mathrm{k}$ and $\mathrm{k}_{1}>=1, \mathrm{k}<=\mathrm{m}$. In this case, the value of the first term of the index related to intra-cluster variance slightly decreases compared to the corresponding term of $D_{1}$ since the clusters in $D_{2}$ are more compact. As a consequence $\operatorname{Scat}(\mathrm{m})<=\operatorname{Scat}(\mathrm{n})$. On the other hand, the second term (inter-cluster density) is increasing as some of the clusters in $D_{1}$ are split and therefore there are borders between clusters that are of high density (for instance clusters 1 and 3 in Fig.c). Then Dens(m) $>$ Dens(n). Based on the above discussion and taking in account that the increase of inter-cluster density is significantly higher than the decrease of intra-cluster variance we may conclude that $S \_D b w_{D 1}<S \_D b w_{D 2}$.

iii) Let $D_{2}$ be a partitioning with less clusters than in $D_{1}(\mathrm{~m}<\mathrm{n})$ and two or more of the clusters in $D_{1}$ are grouped to a cluster in $D_{2}$ (as in Fig.b.). Then, $\exists c_{D 2 j}: c_{D 2 j}=$ $\cup c_{D 1 i}$, where $\mathrm{i}=\mathrm{p}_{1}, \ldots, \mathrm{p}$ and $\mathrm{p}_{1}>=1, \mathrm{p}<=\mathrm{n}$. In this case, the value of the first term of the index related to intra-cluster variance increases as compared to the value of corresponding term of $D_{1}$ since the clusters in $D_{2}$ contain regions of low density. As a consequence, $\operatorname{Scat}(\mathrm{m})>>\operatorname{Scat}(\mathrm{n})$. On the other hand, the second term of the index (inter-cluster density) is slightly decreasing or remains vaguely the same as

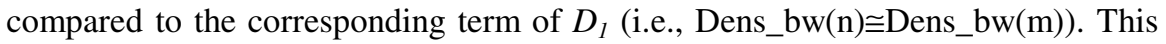
is because similarly to the case of the $D_{l}$ partitioning (Figure $3 \mathrm{a}$ ) there are no borders between clusters in $D_{2}$ that are of high density. Then, based on the above dis- 
cussion and considering that the increase of intra-cluster variance is significantly higher than the decrease of inter-cluster density, we may conclude that $S \_D b w_{D 1}<$ $S \_D b w_{D 2}$.

Lemma 2: Assume a data set $S$ containing convex clusters and a clustering algorithm $A$ applied repetitively to $S$, each time with different parameter values $P_{i}$, resulting in different partitions $D_{i}$ of $S$. For each $D_{i}$ it is true that the correct number of clusters is found. The value $S \_D b w$ is minimized when the optimal partitions are found for the correct number of clusters.

Proof:. We consider $D_{2}$ to be a partitioning with the same number of clusters as the optimal one D1 (Figure 4a), (i.e., $m=n$ ). Furthermore, we assume that one or more of the actual clusters corresponding to $\mathrm{D}_{1}$ are split and their parts are grouped into different clusters in $D_{2}$ (as in Fig. b). That is, if $\mathrm{fC}_{\mathrm{D} 1}=\left\{\mathrm{fc}_{\mathrm{D} 1 \mathrm{p}} \mid \mathrm{p}=1, \ldots, \mathrm{nfr} 1 \mathrm{fc}_{\mathrm{D} 1 \mathrm{p}} \subseteq \mathrm{c}_{\mathrm{D} 1 \mathrm{i}}\right.$, $\mathrm{i}=1, \ldots, \mathrm{n}\}$ a set of clusters fractions in $D_{1}$ then $\exists \mathrm{c}_{\mathrm{D} 2 \mathrm{j}}: \mathrm{c}_{\mathrm{D} 2 \mathrm{j}}=\cup \mathrm{fc}_{\mathrm{D} 1 \mathrm{i}}, \mathrm{i}=\mathrm{p}_{1}, \ldots, \mathrm{p}$ and $\mathrm{p}_{1}>=1, \mathrm{p}<=\mathrm{n}$. In this case, the clusters in $D_{2}$ contain regions of low density (e.g. cluster 1 in Figure $4 \mathrm{~b}$ ) and as a consequence the value of the first term of the index, intracluster variance, increases as compared to the corresponding term of $D_{1}$, i.e., $\operatorname{Scat}(\mathrm{m})>\operatorname{Scat}(\mathrm{n})$. On the other hand, some of the clusters in $D_{2}$ are split and therefore there are border areas between clusters that are of high density (for instance clusters 1 , 3 and 1, 2 in Fig. b). Therefore, the second term (inter-cluster density) of $D_{2}$ is also increasing as compared to the one of $D_{l}$, i.e., Dens_bw $(\mathrm{m})>\operatorname{Dens} \_b w(n)$. Based on the above discussion it is obvious that $S \_D b w_{D 1}<S \_D b w_{D 2}$.

\subsection{Time Complexity}

The complexity of our approach depends on the complexity of the clustering algorithms used for defining the clustering schemes and the complexity of the validity index S_Dbw. More specifically, assume a set of clustering algorithms, $\mathrm{Alg}=\left\{\mathrm{alg}_{1}\right.$, $\left.\ldots, \operatorname{alg}_{\mathrm{k}}\right\}$. Considering the complexity of each of the algorithms, $\mathrm{O}\left(\mathrm{alg}_{\mathrm{i}}\right)$ (where, $\mathrm{i}=1$, $\ldots, \mathrm{k}$ ), we may define the complexity of the whole clustering process, i.e., the process for defining the clustering schemes based on the algorithms under consideration, let $\mathrm{O}(\mathrm{Alg})$. Moreover, the complexity of the index is based on its two terms as defined in (1) and (4). Assuming $d$ is the number of attributes (data set dimension), $c$ is the number of clusters, $n$ is the number of database tuples. The intra-cluster variance complexity is $\mathrm{O}(n d c)$ while the complexity of inter-cluster density is $\mathrm{O}\left(n d c^{2}\right)$. Then S_Dbw complexity is $\mathrm{O}\left(n d c^{2}\right)$. Usually, $c, d<<n$, therefore the complexity of our index for a specific clustering scheme is $\mathrm{O}(n)$. Finally, the complexity of the whole procedure for finding the clustering scheme best fitting a data set, $\mathrm{S}$, among these proposed by the $\mathrm{k}$ algorithms and as a consequence to find the best clustering algorithm is $\mathrm{O}(O(A l g)+n)$.

\section{Experimental Evaluation}

In this section, the proposed approach for selecting the clustering algorithm that results in the optimal clustering scheme for a data set is experimentally tested. In our 
study, we consider three well-known algorithms of different clustering categories, partitional, density-based and hierarchical (K-means, DBSCAN and CURE respectively). Also, we experiment with real and synthetic multidimensional data sets containing different number of clusters. In the sequel, due to lack of space, we present only some representative examples of our experimental study.

We consider two 2-dimensional data sets containing four and seven clusters respectively as Fig. 5, Fig. 6 depict. Applying the above mentioned clustering algorithms (i.e., K-means, DBSCAN and CURE) to these data sets three sets of clustering schemes are produced. Each of the clustering schemes' sets corresponds to the clustering results of an algorithm for different values of its input parameters. Then we evaluate the defined clustering schemes based on the proposed index, S_Dbw. The clustering scheme at which S_Dbw is minimized indicates the best partitioning of data set and as a consequence the best algorithm and its input parameters' values resulting in it.

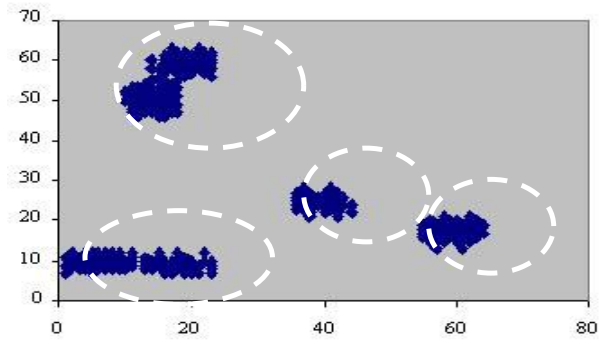

Fig. 5. DataSet1 - A Synthetic Data Set containing four clusters
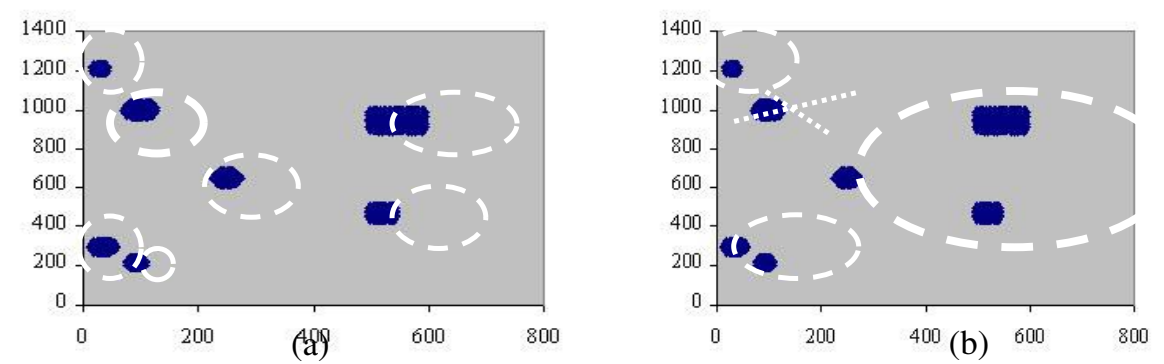

Fig. 6. DataSet2 - A partitioning of data set into seven clusters using (a) CURE or DBSCAN, (b) K-Means

Table 1 and Table 2 summarize the values of S_Dbw in each of the above cases. More specifically, Table 1 depicts that S_Dbw takes its minimum value when DataSet1 is partitioned into four clusters (i.e., actual clusters in data set) no matter which algorithm is used. This means that according to our approach the optimal partitioning of DataSet1 is four clusters and all three algorithms partitioned it in a right way (i.e., they find the actual clusters in the data set). Figure 5 is a visualization of DataSet1 while the cycles indicate the proposed partitioning. 
Table 1. The values of SD_bw for DataSet1clustering schemes

\begin{tabular}{|c|c|c|c|c|c|c|}
\hline & \multicolumn{2}{|c|}{ K-means } & \multicolumn{2}{|c|}{ DBSCAN } & \multicolumn{2}{|c|}{$\begin{array}{c}\text { CURE } \\
\mathrm{r}=10, \mathrm{a}=0.3\end{array}$} \\
\hline $\begin{array}{l}\begin{array}{l}\text { No } \\
\text { clusters }\end{array} \\
\end{array}$ & Input & $\begin{array}{l}\text { S_Dbw } \\
\text { Value }\end{array}$ & Input & $\begin{array}{l}\text { S_Dbw } \\
\text { Value }\end{array}$ & Input & $\begin{array}{l}\text { S_Dbw } \\
\text { Value }\end{array}$ \\
\hline 8 & $\mathrm{C}=8$ & 0.124 & & - & $\mathrm{C}=8$ & 0.108 \\
\hline 7 & $\mathrm{C}=7$ & 0.118 & & - & $\mathrm{C}=7$ & 0.103 \\
\hline 6 & $\mathrm{C}=6$ & 0.0712 & Eps $=20$ MinPts $=4$ & 0.087 & $\mathrm{C}=6$ & 0.082 \\
\hline 5 & $\mathrm{C}=5$ & 0.086 & Eps $=10$ MinPts $=10$ & 0.086 & $\mathrm{C}=5$ & 0.091 \\
\hline 4 & $\mathrm{C}=4$ & 0.0104 & Eps $=40$ MinPts $=10$ & 0.0104 & $\mathrm{C}=4$ & 0.0104 \\
\hline 3 & $\overline{\mathrm{C}=3}$ & 0.0312 & Eps $=10$ MinPts $=15$ & 0.031 & $\overline{\mathrm{C}=3}$ & 0.031 \\
\hline$\underline{2}$ & $\mathrm{C}=2$ & 0.1262 & Eps $=20$ MinPts $=15$ & 0.1262 & $\mathrm{C}=2$ & 0.126 \\
\hline
\end{tabular}

Table 2. The values of SD_bw for DataSet 2 clustering schemes

\begin{tabular}{|c|c|c|c|c|c|c|}
\cline { 2 - 7 } \multicolumn{1}{c|}{} & \multicolumn{2}{c|}{ K-means } & \multicolumn{2}{c|}{ DBSCAN } & \multicolumn{2}{c|}{$\begin{array}{c}\text { CURE } \\
\mathrm{r}=10, \mathrm{a}=0.3\end{array}$} \\
\hline $\begin{array}{c}\text { No } \\
\text { clusters }\end{array}$ & Input & $\begin{array}{c}\text { S_Dbw } \\
\text { Value }\end{array}$ & Input & $\begin{array}{l}\text { S_Dbw } \\
\text { Value }\end{array}$ & Input & $\begin{array}{c}\text { S_Dbw } \\
\text { Value }\end{array}$ \\
\hline 8 & $\mathrm{C}=8$ & 0.66 & Eps=8 MinPts=10 & 0.0333 & $\mathrm{C}=8$ & 0.0517 \\
\hline 7 & $\mathrm{C}=7$ & 0.6004 & $\underline{\mathbf{E p s}=\mathbf{2 0}}$ & $\underline{\mathbf{0 . 0 0 0 9}}$ & $\underline{\mathbf{C}=7}$ & $\underline{\mathbf{0 . 0 0 0 9}}$ \\
\hline 6 & $\mathrm{C}=6$ & 0.575 & Eps=80 MinPts=4 & 0.0018 & $\mathrm{C}=6$ & 0.0019 \\
\hline 5 & $\mathrm{C}=5$ & 0.491 & & - & $\mathrm{C}=5$ & 0.0051 \\
\hline 4 & $\mathrm{C}=4$ & 0.365 & & - & $\mathrm{C}=4$ & 0.032 \\
\hline 3 & $\mathrm{C}=3$ & 0.045 & & - & $\mathrm{C}=3$ & 0.073 \\
\hline$\underline{2}$ & $\mathrm{C}=2$ & 0.854 & & - & $\mathrm{C}=2$ & 0.796 \\
\hline
\end{tabular}

Moreover, according to Table 2, in case of DataSet2, S_Dbw takes its minimum value for the partitioning of seven clusters defined by DBSCAN and CURE. This is also the number of actual clusters in the data set. Fig. a presents the partitioning of Dataset2 into seven clusters as defined by DBSCAN and CURE while the clustering result of K-Means into seven clusters is presented in Fig. b. It is obvious that K-Means fails to partition DataSet2 properly even in case that the correct number of clusters (i.e., c=7) is considered.

The value of our approach is more evident in case of multidimensional data sets where efficient visualization is difficult or even impossible. We consider two synthetic data sets, four- and six-dimensional (further referred as DataSet3 and DataSet4 respectively). We may discover four clusters in DataSet3 while the number of clusters occurred in DataSet4 is two. Assuming DataSet3 our approach proposes the partitioning of four clusters defined by DBSCAN and CURE as the best fitting the data under consideration. Four clusters is the value at which S_Dbw takes its minimum value (see Table 3) and it is also the actual number of clusters in data set. Similarly, in case of DataSet4 SD_bw takes its minimum value for the partitioning of two clusters defined by DBSCAN (see Table 4). Considering the clustering schemes proposed by CURE, S_Dbw is minimized when $\mathrm{c}=5$. Thus, the best partitioning among these defined by CURE is five clusters. It is obvious that CURE seems to fail to partition Dataset 4 in a right way, even in case that the actual number of clusters (i.e., two) is considered. 
Table 3. The values of S_Dbw for 4D-data set clustering schemes

\begin{tabular}{|c|c|c|c|c|c|c|}
\hline \multirow[b]{2}{*}{$\begin{array}{l}\text { No } \\
\text { clus- } \\
\text { ters }\end{array}$} & \multicolumn{2}{|r|}{ K-means } & \multicolumn{2}{|l|}{ DBSCAN } & \multicolumn{2}{|c|}{$\begin{array}{c}\text { CURE } \\
\mathrm{r}=10, \mathrm{a}=0.3\end{array}$} \\
\hline & Input & S_Dbw Value & Input & $\begin{array}{l}\text { S_Dbw } \\
\text { Value }\end{array}$ & Input & $\begin{array}{c}\text { S_Dbw } \\
\text { Value }\end{array}$ \\
\hline 9 & $\mathrm{C}=9$ & $2.3555556727820735 \mathrm{E} 7$ & Eps $=15$ MinPts $=4$ & 0.042 & $\mathrm{C}=9$ & 0.2739 \\
\hline 8 & $\mathrm{C}=8$ & 0.618 & Eps $=15$ MinPts $=10$ & 0.226 & $\mathrm{C}=8$ & 0.256 \\
\hline 7 & $\mathrm{C}=7$ & 1.295 & - & - & $\mathrm{C}=7$ & 0.1765 \\
\hline 6 & $\mathrm{C}=6$ & 1800005.464 & $\mathrm{Eps}=20 \mathrm{MinPts}=4$ & 0.0365 & $\mathrm{C}=6$ & 0.1899 \\
\hline 5 & $\mathrm{C}=5$ & 1.02165327 & Eps $=10$ MinPts $=10$ & 0.0311 & $\mathrm{C}=5$ & 0.0859 \\
\hline 4 & $\mathrm{C}=4$ & 295333334.99 & Eps $=40$ MinPts $=10$ & 0.0013 & $\mathrm{C}=4$ & 0.0013 \\
\hline 3 & $\mathrm{C}=3$ & 1.031 & & - & $\mathrm{C}=3$ & 0.0149 \\
\hline 2 & $\mathrm{C}=2$ & 4.197 & & - & $\mathrm{C}=2$ & 0.672 \\
\hline
\end{tabular}

Table 4. The values of SD_bw for the 6D-data set clustering schemes

\begin{tabular}{|c|c|c|c|c|c|c|}
\cline { 2 - 7 } \multicolumn{1}{c|}{} & \multicolumn{2}{c|}{ K-means } & \multicolumn{2}{c|}{ DBSCAN } & \multicolumn{2}{c|}{$\begin{array}{c}\text { CURE } \\
\mathrm{r}=10, \mathrm{a}=0.3\end{array}$} \\
\hline No clusters & Input & S_Dbw Value & Input & S_Dbw Value & Input & $\begin{array}{c}\text { S_Dbw } \\
\text { Value }\end{array}$ \\
\hline 8 & $\mathrm{C}=8$ & 0.689 & - & - & $\mathrm{C}=8$ & 0.291 \\
\hline 7 & $\mathrm{C}=7$ & 0.653 & - & - & $\mathrm{C}=7$ & 0.338 \\
\hline 6 & $\mathrm{C}=6$ & 0.662 & - & - & $\mathrm{C}=5$ & 0.322 \\
\hline 5 & $\mathrm{C}=5$ & 0.669 & - & 0.239 \\
\hline 4 & $\mathrm{C}=4$ & 0.58 & $\mathrm{Eps}=5$ MinPts=4 & 0.31 & $\mathrm{C}=4$ & 0.805 \\
\hline 3 & $\mathrm{C}=3$ & 0.619 & $\mathrm{Eps}=25 \mathrm{MinPts}=4$ & 0.233 & $\mathrm{C}=3$ & 1.401 \\
\hline $\mathbf{2}$ & $\mathrm{C}=2$ & 0.114 & $\underline{\text { Eps=35 MinPts=4 }}$ & $\underline{\mathbf{0 . 0 9 6}}$ & $\mathrm{C}=2$ & 2.3249 \\
\hline
\end{tabular}

Finally, we evaluate our approach using real data sets. One of the data sets, we studied, contains three parts of Greek roads network [17]. The roads are represented by their MBR approximations' vertices. Figure 7 is a visualization of this data. The

Table 5. The values of SD_bw for Real_Data1 (Figure 7)

\begin{tabular}{|c|c|c|c|c|c|c|}
\cline { 2 - 7 } \multicolumn{1}{c|}{} & \multicolumn{2}{c|}{ K-means } & \multicolumn{2}{c|}{ DBSCAN } & \multicolumn{2}{c|}{$\begin{array}{c}\text { CURE } \\
\mathrm{r}=10, \mathrm{a}=0.3\end{array}$} \\
\hline $\begin{array}{c}\text { No } \\
\text { Clusters }\end{array}$ & Input & S_Dbw Value & Input & S_Dbw Value & Input & $\begin{array}{c}\text { S_Dbw } \\
\text { Value }\end{array}$ \\
\hline 8 & $\mathrm{C}=8$ & 0.179 & & - & $\mathrm{C}=8$ & 0.1694 \\
\hline 7 & $\mathrm{C}=7$ & 0.237 & & - & $\mathrm{C}=7$ & 0.1914 \\
\hline 6 & $\mathrm{C}=6$ & 0.343 & & - & $\mathrm{C}=6$ & 0.2248 \\
\hline 5 & $\mathrm{C}=5$ & 0.367 & & - & $\mathrm{C}=5$ & 0.1621 \\
\hline 4 & $\mathrm{C}=4$ & 0.35 & Eps=50000 MinPts=10 & 0.192 & $\mathrm{C}=4$ & 0.1349 \\
\hline $\mathbf{3}$ & $\underline{\mathbf{C}=\mathbf{3}}$ & $\underline{\mathbf{0 . 0 8 3}}$ & $\underline{\text { Eps=30000 MinPts=10 }}$ & $\underline{\mathbf{0 . 0 8 4}}$ & $\mathrm{C}=3$ & 0.1086 \\
\hline 2 & $\mathrm{C}=2$ & 0.918 & Eps=10000 MinPts=10 & 0.891 & $\mathrm{C}=2$ & 1.0508 \\
\hline
\end{tabular}

behaviour of S_Dbw regarding the different clustering schemes (i.e., number of clusters) defined by the above mentioned algorithms are depicted in Table 5. It is clear that S_Dbw indicates the correct number of clusters (three) as the best partitioning for the data set when K-Means or DBSCAN is used. 


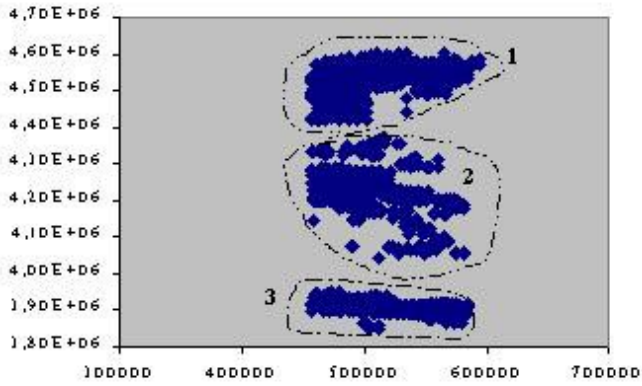

Fig. 7. Real_Data1-A data set representing a part of Greek network

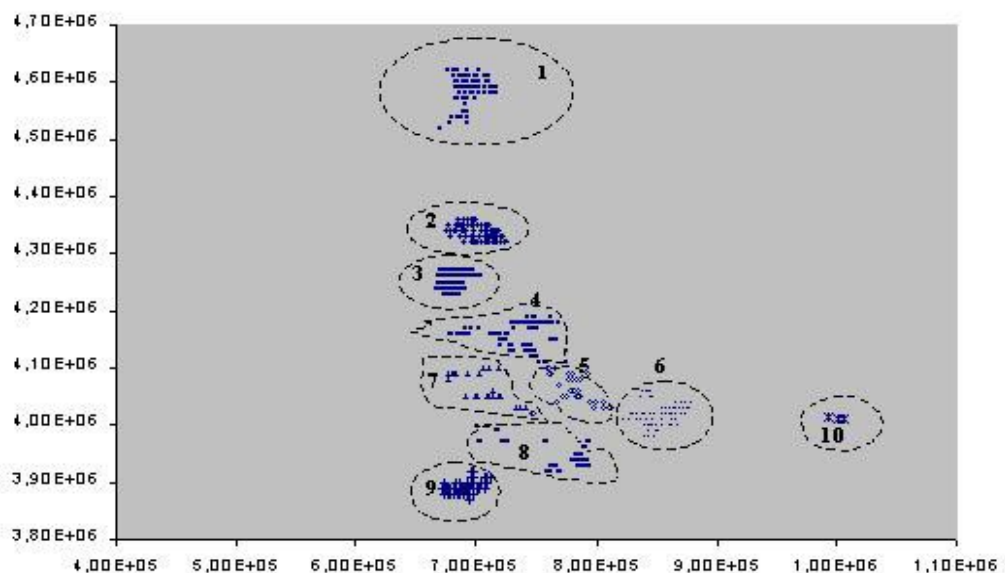

Fig. 8. Real_Data2 - A data set representing towns and villages of Greek islands

Table 6. The values of SD_bw for Real_Data2 (Figure 8)

\begin{tabular}{|l|l|l|l|l|l|l|}
\cline { 2 - 7 } \multicolumn{1}{c|}{} & \multicolumn{2}{c|}{ K-means } & \multicolumn{2}{c|}{ DBSCAN } & \multicolumn{2}{c|}{$\begin{array}{c}\text { CURE } \\
\mathrm{r}=10, \mathrm{a}=0.3\end{array}$} \\
\hline $\begin{array}{c}\text { No } \\
\text { Clusters }\end{array}$ & Input & S_Dbw Value & \multicolumn{1}{c|}{ Input } & S_Dbw Value & Input & S_DbwValue \\
\hline 11 & $\mathrm{C}=11$ & 0.039 & - & - & $\mathrm{C}=11$ & 0.114 \\
\hline $\mathbf{1 0}$ & $\mathbf{C}=\mathbf{1 0}$ & $\mathbf{0 . 0 3 4 8}$ & Eps=2000, MinPts=4 & 0.113 & $\mathrm{C}=10$ & 0.08 \\
\hline 9 & $\mathrm{C}=9$ & 0.0503 & Eps=2000 MinPts=10 & 0.072 & $\mathrm{C}=9$ & 0.049 \\
\hline 8 & $\mathrm{C}=8$ & 0.088 & $\mathrm{Eps}=3000$ MinPts=4 & 0.123 & $\mathrm{C}=8$ & 0.107 \\
\hline 7 & $\mathrm{C}=7$ & 0.112 & - & - & $\mathrm{C}=7$ & 0.121 \\
\hline 6 & $\mathrm{C}=6$ & 0.095 & Eps=4000 MinPts=4 & 0.228 & $\mathrm{C}=6$ & 0.32 \\
\hline 5 & $\mathrm{C}=5$ & 0.149 & - & - & $\mathrm{C}=5$ & 0.266 \\
\hline 4 & $\mathrm{C}=4$ & 0.621 & Eps=5000 MinPts=4 & 0.561 & $\mathrm{C}=4$ & 0.511 \\
\hline 3 & $\mathrm{C}=3$ & 0.539 & $\mathrm{Eps}=6000$ MinPts=4 & 0.251 & $\mathrm{C}=3$ & 0.389 \\
\hline 2 & $\mathrm{C}=2$ & 1.150 & $\mathrm{Eps}=7000$ MinPts=4 & 0.621 & $\mathrm{C}=2$ & 0.617 \\
\hline
\end{tabular}


We carried out, a similar experiment using a data set representing the towns and villages of a group of Greek islands [17]. A visualization of this data is presented in Figure 8. Based on Table 6, we observe that SD_bw takes its minimum value for the clustering scheme of ten clusters as defined by K-Means, which is a "good" approximation of the inherent clusters in underlying data. Both CURE and DBSCAN fail to define a good partitioning for the data under consideration.

Then, it is clear that S_Dbw can assist to select the clustering algorithm resulting in the optimal partitioning of the data set under consideration as well as the input parameters' values of the selected algorithm based on which the optimal partitioning is defined.

\section{Conclusions}

Clustering algorithms may result in different clustering schemes under the consideration of different assumption. Moreover, there are cases that a clustering algorithm may partition a data set into the correct number of clusters but in a wrong way. In most of the cases the users visually verify the clustering results. In the case of voluminous and/or multidimensional data sets where efficient visualization is difficult or even impossible, it becomes tedious to know if the results of clustering are valid or not.

In this paper we addressed the important issue of assessing the validity of clustering algorithms' results, so as to select the algorithm and its parameters values for which the optimal partitioning of a data set is defined (assuming that the data set presents clustering tendency). We propose a clustering validity approach based on a new validity index (S_Dbw) for assessing the results of clustering algorithms. The index is optimised for data sets that include compact and well-separated clusters. The compactness of the data set is measured by the cluster variance where as the separation by the density between clusters.

We have proved our approach reliability and value i. theoretically, by illustrating the intuition behind it and ii. experimentally, using various data sets of non-standard (but in general non-convex) geometries covering also the multidimensional case. The index, as indicated by experiments, may always indicate the algorithm and the respective input parameters' values so as to find the inherent clusters for a data set.

Further Work. As we mentioned earlier the validity assessment index we proposed in this paper works better when the clusters are mostly compact. It does not work properly in the case of clusters of non-convex (i.e., rings) or extraordinarily curved geometry. We are going to work on this issue as the density and its continuity is not any more sufficient criteria. We plan an extension of this effort to be directed towards an integrated algorithm for cluster discovery putting emphasis on the geometric features of clusters, using sets of representative points, or even multidimensional curves rather than a single center point.

Acknowledgements. This work was supported by the General Secretariat for Research and Technology through the PENED ("99\#\$ 85") project. We thank Y. Batistakis for his help in the experimental study. We are also grateful to C. Rodopoulos and C. Amanatidis for the implementation of CURE algorithm as well as to Drs Joerg Sander and Eui-Hong (Sam) Han for providing information and the source code for DBSCAN and CURE algorithms respectively. 


\section{References}

1. Rakesh Agrawal, Johannes Gehrke, Dimitrios Gunopulos, Prabhakar Raghavan, "Automatic Subspace Clustering of High Dimensional Data for Data Mining Applications", Proceedings of SIGMOD, 1998.

2. Michael J. A. Berry, Gordon Linoff. Data Mining Techniques For marketing, Sales and Customer Support. John Willey \& Sons, Inc, 1996.

3. Martin Ester, Hans-Peter Kriegel, Jorg Sander, Michael Wimmer, Xiaowei Xu. "Incremental Clustering for Mining in a Data Warehousing Environment", Proceedings of $24^{\text {th }}$ VLDB Conference, New York, USA, 1998.

4. Martin Ester, Hans-Peter Kriegel, Jorg Sander, Xiaowei Xu. "A Density-Based Algorithm for Discovering Clusters in Large Spatial Databases with Noise", Proceedings of $2^{\text {nd }}$ Int. Conf. On Knowledge Discovery and Data Mining, Portland, OR, pp. 226-231, 1996.

5. Usama M. Fayyad, Gregory Piatesky-Shapiro, Padhraic Smuth and Ramasamy Uthurusamy. Advances in Knowledge Discovery and Data Mining. AAAI Press 1996

6. Sudipto Guha, Rajeev Rastogi, Kyueseok Shim. "CURE: An Efficient Clustering Algorithm for Large Databases", Published in the Proceedings of the ACM SIGMOD Conference, 1998.

7. Sudipto Guha, Rajeev Rastogi, Kyueseok Shim. "ROCK: A Robust Clustering Algorithm for Categorical Attributes", Published in the Proceedings of the IEEE Conference on Data Engineering, 1999.

8. Alexander Hinneburg, Daniel Keim. "An Efficient Approach to Clustering in Large Multimedia Databases with Noise". Proceeding of KDD '98, 1998.

9. A.K Jain, M.N. Murty, P.J. Flyn. "Data Clustering: A Review", ACM Computing Surveys, Vol. 31, No3, September 1999.

10. Milligan, G.W. and Cooper, M.C. (1985), "An Examination of Procedures for Determining the Number of Clusters in a Data Set", Psychometrika, 50, 159-179.

11. Raymond Ng, Jiawei Han. "Efficient and Effective Clustering Methods for Spatial Data Mining". Proceeding of the $20^{\text {th }}$ VLDB Conference, Santiago, Chile, 1994.

12. C. Sheikholeslami, S. Chatterjee, A. Zhang. "WaveCluster: A-MultiResolution Clustering Approach for Very Large Spatial Database". Proceedings of $24^{\text {th }}$ VLDB Conference, New York, USA, 1998.

13. Sharma S.C. Applied Multivariate Techniques. John Willwy \& Sons, 1996.

14. S. Theodoridis, K. Koutroubas. Pattern recognition, Academic Press, 1999

15. M. Halkidi, M. Vazirgiannis, I. Batistakis. "Quality scheme assessment in the clustering process", In Proceedings of PKDD, Lyon, France, 2000.

16. Ramze Rezaee, B.P.F. Lelieveldt, J.H.C Reiber. "A new cluster validity index for the fuzzy c-mean", Pattern Recognition Letters, 19, pp237-246, 1998.

17. Y. Theodoridis. Spatial Datasets: an "unofficial" collection. http://dias.cti.gr/ ytheod/research/datasets/spatial.html 